This item was submitted to Loughborough's Institutional Repository (https://dspace.lboro.ac.uk/) by the author and is made available under the following Creative Commons Licence conditions.

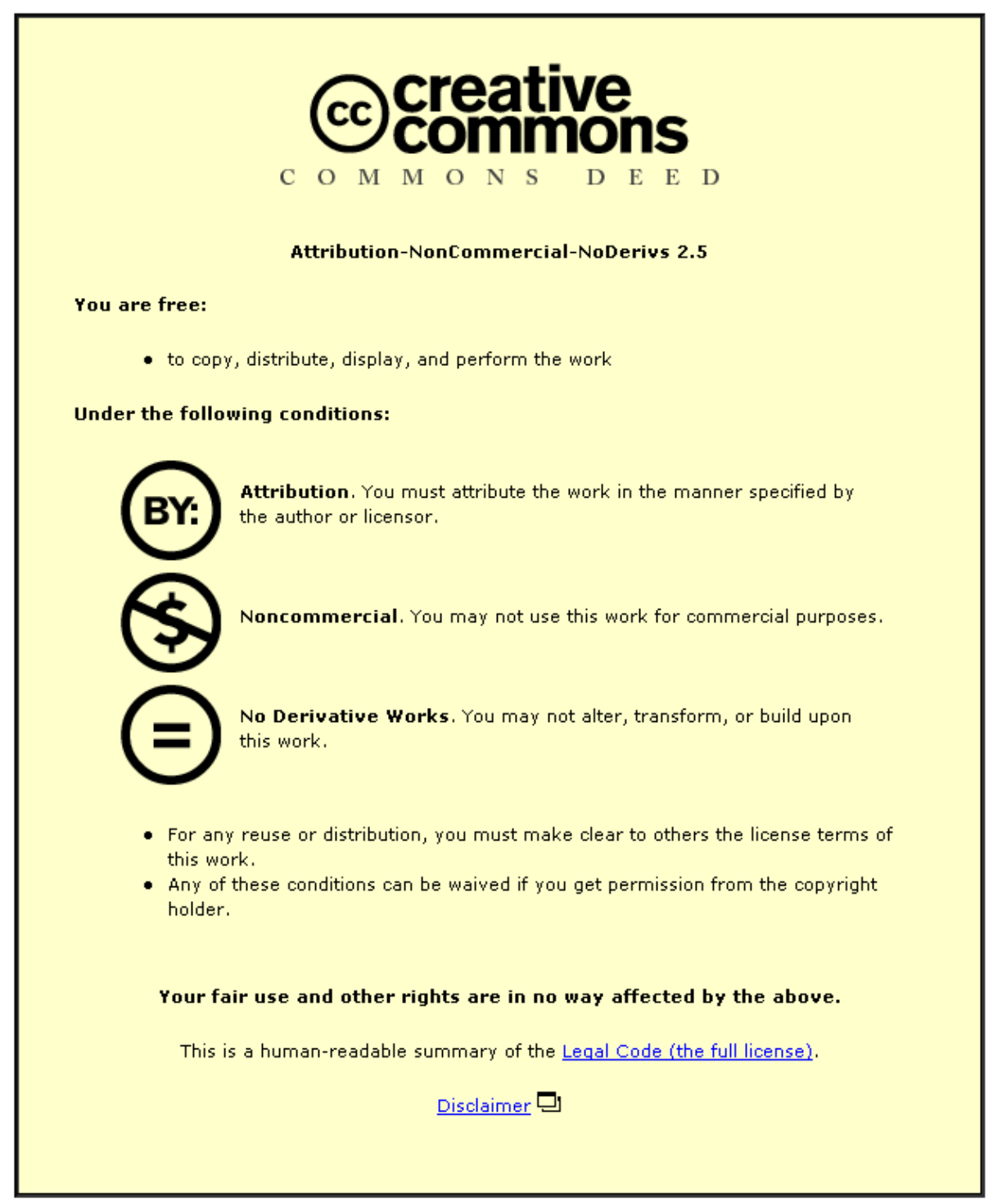

For the full text of this licence, please go to: http://creativecommons.org/licenses/by-nc-nd/2.5/ 


\title{
Performance Metrics for the Prediction of Link and Path Availability in VANETs
}

\author{
Zulkefli M. Yusof ${ }^{1}$ and J.A. Flint ${ }^{2}$ \\ Department of Electronic and Electrical Engineering \\ Loughborough University \\ LE11 3TU, UK \\ 1 elzy@lboro.ac.uk \\ 2j.a.flint@lboro.ac.uk
}

\begin{abstract}
In this paper metrics are produced which may be used for performance enhancement in Vehicular ad-hoc networks (VANETs) protocols. The method described makes use of the concept of link availability estimates which are produced on an individual link basis and which may be combined by multiplication to produce a quality metric for the whole route. The technique is discussed in the context of the Ad hoc On-demand Distance Vector (AODV) routing method.
\end{abstract}

Keywords - Performance Metrics; Link and Path Availability; VANETs; AODV; NS2

\section{INTRODUCTION}

The characteristics of Vehicular ad-hoc networks [1] (VANETs) have always made them an interesting topic for research. There are numerous possibilities for exploiting the specific behaviour observed to enhancing performances, and in addition there are many levels within the OSI model where this can be done, including the possibility to make use of multiple layers, or cross-layers. This paper will mainly address the network layer where a routing protocol can be enhanced for improving performance by better maintenance links in a route. The main concept introduced is the proposed use of link availability as a metric, which is estimated from positional and velocity vector data at the nodes, rather than being determined absolutely. This metric can be used to more accurately determine a good link than conventional techniques.

The paper starts by giving an introduction to VANETs, routing protocol targeted by the metrics developed in this paper, and some other relevant works. This is followed by location predictions, implementation framework, a brief simulation validation example, and finally the conclusion.

\section{A. VANET}

VANETs are in a specific class or subset of Mobile Ad hoc Networks (MANETs) [2]. They are characterized by being self organizing communication networks built up of moving nodes (vehicles) with very high mobility and limited degrees of freedom in the mobility process. In other words, rather than moving completely at random, vehicles tend to move in an organized fashion though some random processes do take place. Most vehicles are restricted in their range of motion, for example by being constrained to follow single or multilane highways.

There are also many types of routing protocols available for ad hoc networks. The protocols are usually categorized as Proactive, Reactive, [3] and Hybrid which combines the first two categories. The dynamic nature of VANETs makes proactive protocols not the obvious choice since it is challenging to maintain the routing table with predetermined routes. Reactive protocols are however widespread due to their simple implementation, however they have limitations which make a hybrid approach preferable. For a hybrid approach to work successfully it must exploit performance metrics of some sort to predict the likely link quality. The objective of the paper is therefore to develop metrics to assist the pre-emptive part of a hybrid routing algorithm.

\section{B. AODV Routing Protocol}

Ad Hoc On-Demand Distance-Vector Routing (AODV) [4] is the routing protocol chosen by means of an example in this study. It provides communication between mobile nodes with minimal control overhead and does not maintain routes from every node to every other node in the network. Routes are discovered on an ad hoc basis and maintained only for as long as they are necessary. It is loop-free because it increases the sequence number each time it learns of any change in the topology of its neighbours. The sequence number ensures that the most recent route is selected for a route discovery.

Control packets are used in the AODV protocol to establish link, provides data transmission, and also maintenance. Route Request (RREQ) packet is used to initiate the route discovery process. Route Reply (RREP) is used to finalize a route. Route Error (RERR) packet is used when there is a link break.

The AODV process description starts with a node, called the source node (SN) which begins to execute packet transmission to a destination node (DN). Any node involves in creating a link will first look at its own routing table to see 
if the $\mathrm{DN}$ is in the routing table. Referring to the flow chart in Fig. 1, it is assumed that the DN is not in the table and therefore starts with SN broadcasting RREQ packets to its neighbouring nodes.

The node which received the RREQ packet will see if it is the destination by checking the IP address in the packet. If it is not the DN, the node adds the route reverse entry in its routing table to be used as a route for the reply packet from $\mathrm{DN}$ and data packet by the SN. The forwarding of packet continues until it reaches the $\mathrm{DN}$, and if it fails to be delivered after a specified time the packet will be dropped.

As the RREQ packet reaches the destination, the DN will acknowledge by replying a RREP packet which will go to the $\mathrm{SN}$ via the reverse route. Upon receiving the RREP packet, the SN starts the transmission by forwarding the data packets via the reverse route entry in routing table of each node in the route.

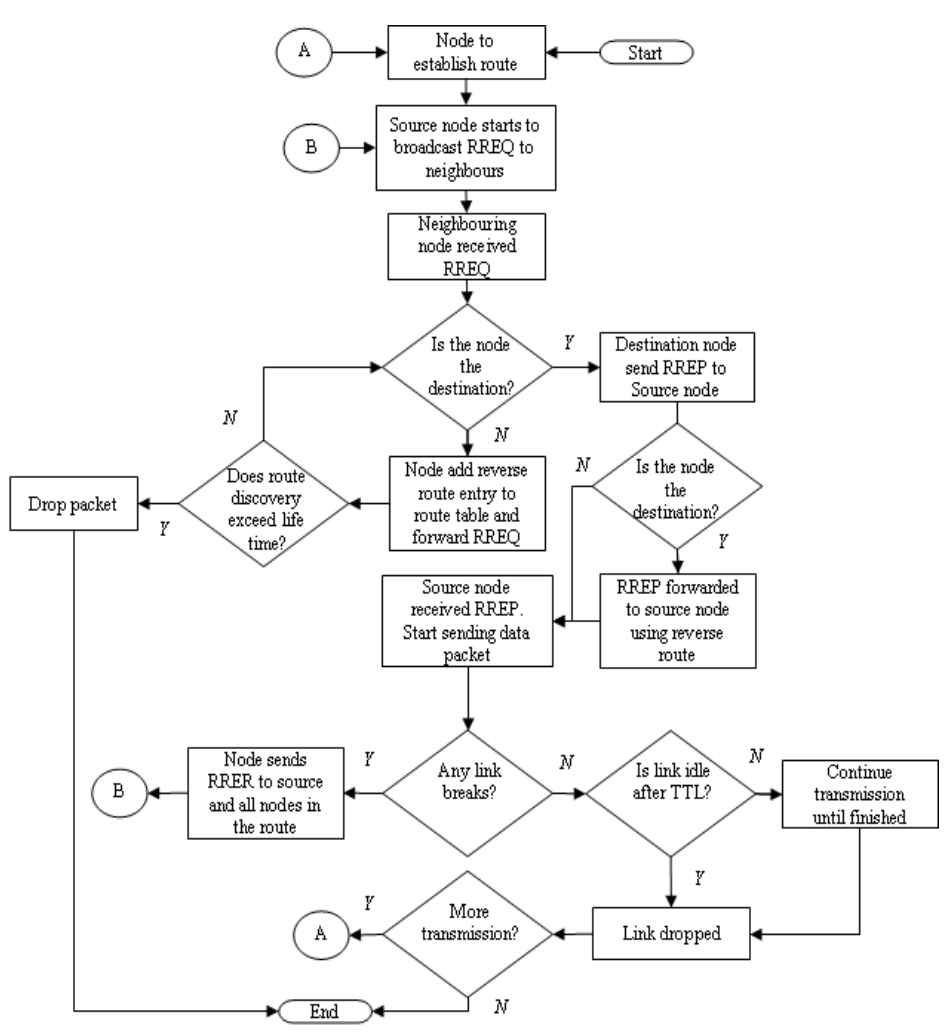

Fig. 1. The AODV process flow chart

A 'hello' packet continues to be periodically broadcasted even when a route has been established and communication between $\mathrm{SN}$ and DN is in progress to ensure that a link is still available or active. If a transmission is completed and the route is sensed to be idling after a specified time then the link is dropped. Whenever a link is broken by the way of idling, or by the detection of using Hello packets, or by the notification from the MAC layer, the RREQ packets will be issued by the surviving node to its neighbours downstream and eventually reaching the $\mathrm{SN}$ to inform that the link is broken. In a situation if the link break is near the DN, a local repair will be carried out. The node will create a buffer to store the incoming data packets will trying to re-establish link and resume if it is successful, or otherwise link is dropped and RREQ is issued.

Illustrating the process, as an example Fig. 2 shows a source node initiates the route discovery by broadcasting RREQ packets to its neighbours. Each nodes $A_{1}, B_{1}$, and $E_{1}$ receives the packet and look up at each own routing table to see if it is the destination node. As it is not, each node updates its routing table with a reverse route entry to be used for both RREP to finalize the discovery process and also for the data transmission. The node forwards the RREQ packet to its neighbour, repeating the same process described earlier until it reaches the destination. In this example, the scenario applies to the route with the $B_{n}$ nodes.

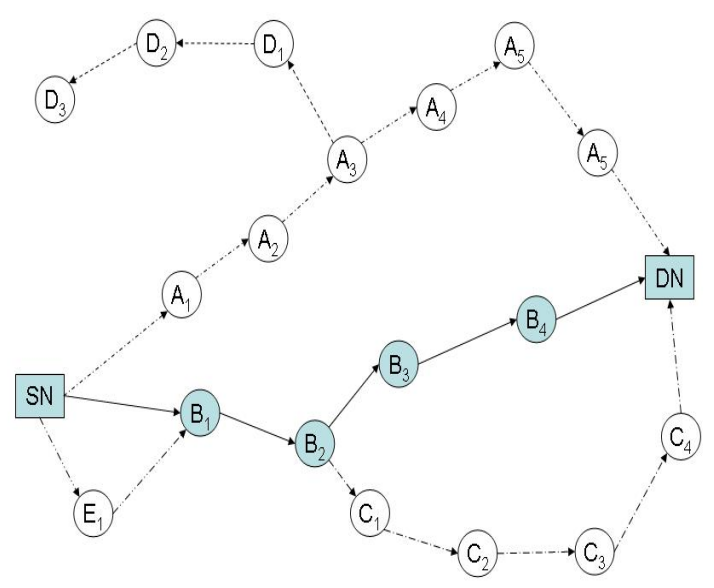

Fig. 2. Scenario illustrating the AODV process

\section{C. $\quad$ Other Works}

A pre-emptive algorithm was proposed by Goff et al. [5] where the estimation of the pre-emptive region is based on the signal power threshold under ideal conditions. Menouar et al. [6] proposed a movement prediction-based routing concept for VANETs called MOvement Prediction-based Routing (MOPR) which predicts the future position and link lifetime estimation of vehicles to avoid link rupture. It considers path stability and link stability in its calculation by using the Pythagorean Theorem where a link state is the lifetime over the maximum lifetime and assumes the local availability of a neighbouring node's movement information. In [7] shows that pre-emptive action is taken when the neighbour's signal strength ratio of received power to receive threshold power is not less than 1. Additions were made to 
the AODV protocol by adding tables applied by each node, and the modification on the 'hello' packets.

Vo and Hong [8] used the hop count metric to discover the availability of multiple paths, although hop count alone does not take the packet loss or bandwidth into consideration, which is likely to result in low throughput along the shortest path. When combined with a bandwidth estimation technique at each node, the node is able to predict the risk of congestion over its connected links and to select the high available bandwidth link for forwarding packets. Klem et al. [9] have proposed a link management protocol that uses signal strength measurements, which look into the frequent link failures caused by the nodes mobility. When there is an indication that the node is likely to be out of range, it reacts by using a temporarily higher transmission power to keep the link alive. If there is a possibility that the link is about to fail, it proactively initiates a route rediscovery procedure. Changes were also made to the MAC layer to avoid false link failure caused by congestion. Chen et al. [10] proposed a simulation-based method, based on a road network model using graphs of cellular automata to predict the future trajectories of moving objects. Chao and Aiqun [11] showed improvement can be made by reducing the message overhead. This involves enhancing the method by which 'hello' packets are sent by changing the interval dynamically by referring to the link availability. McDonald and Znati [12] proposed a routing metric which defines probabilistic measures of the availability of network paths that are subject to link failures caused by node mobility and use it to select a more stable path and reduce the routing overheads. It comprises of a mobility model and a link availability mode. Each node has a movement which consists of a sequence of random length intervals called mobility epochs during which the node moves in a constant direction. The link availability relies heavily on a probabilistic model to predict the future status of the link.

The current paper offers alternative approach whereby the availability is estimated by making use of position vectors of the nodes and their velocities.

\section{LOCATION PREDICTION}

This section explains how the alternative routes are identified. Later, the methods to calculate link availability and the selection of best alternative route are presented. The assumptions are: (a) all nodes are equipped with GPS units or similar and can determine their position at any given time, (b) the two nodes in a given potential link are aware of each others' coordinates and velocity vectors.

\section{A. Pre-defining Alternative Routes}

This process provides the estimation that predicts how long a two-node link takes to go out of range of each other in seconds. Referring to the information from the routing table the location is then applied on a Cartesian coordinate system where the nodes move in a vector of $\mathrm{x}$ and $\mathrm{y}$ along the $\mathrm{x}$ and $\mathrm{y}$ axes respectively. Using the Pythagorean Theorem, the Euclidean distance can be derived as shown in Fig. 3 where $\left(\mathrm{x}_{0}, \mathrm{y}_{0}\right)$ and $\left(\mathrm{x}_{1}, \mathrm{y}_{1}\right)$ are points in the plane with the inclusion of time and velocity in the equation so that the time when the distance between the two nodes greater than the transmission range can be computed.

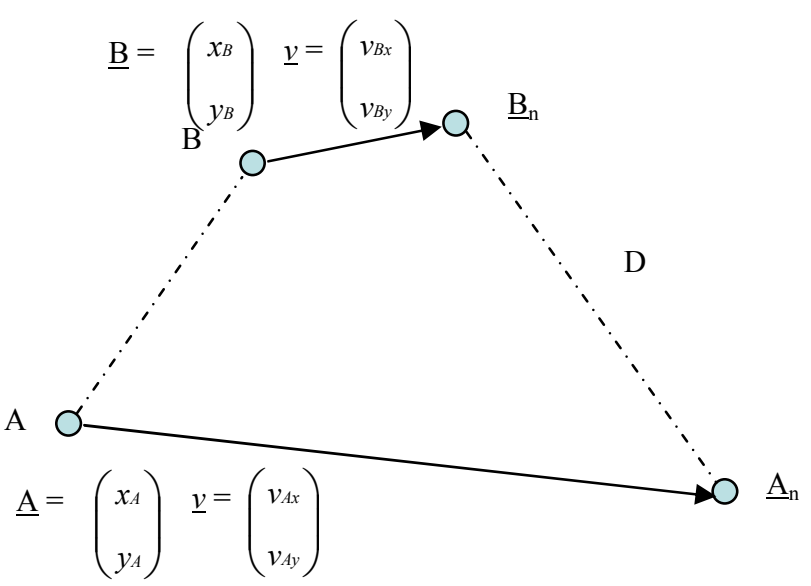

Fig. 3. Projection of node movement

Node 1 starts from location $\mathrm{x}_{1}, \mathrm{y}_{1}$ and node 2 from position $\mathrm{x}_{2}, \mathrm{y}_{2}$. Each node moves with a known velocity vector which is assumed constant during the calculation.

Position of $A$ and $B$ after the time $n \Delta t$, where $n$ is step number and $\Delta \mathrm{t}$ is step length,

$$
\mathrm{A}_{\mathrm{n}}=\mathrm{A}_{0}+\mathrm{v}_{\mathrm{A}} \cdot \mathrm{n} \Delta \mathrm{t}, \quad \mathrm{B}_{\mathrm{n}}=\mathrm{B}_{0}+\mathrm{v}_{\mathrm{B}} \cdot \mathrm{n} \Delta \mathrm{t}
$$

$\left|A_{n} B_{n}\right|=D$, which is the distance between two nodes in a link calculated and used to determine if the link is out of the transmission range, and $t$ defines the link life time which is defined as the time it takes from the link being first initiated, $\mathrm{t}_{0}$, until it reaches the location where the distance between the two nodes (D) reaches the transmission range $(R) t_{1}$.

$$
\begin{aligned}
& \mathrm{A}_{\mathrm{n}}=\left(\begin{array}{c}
x_{A 0}+v_{a x} . n \Delta t \\
y_{A 0}+v_{a y} . n \Delta t
\end{array}\right) \quad \mathrm{B}_{\mathrm{n}}=\left(\begin{array}{c}
x_{B 0}+v_{B x} . n \Delta t \\
y_{B 0}+v_{B y} . n \Delta t
\end{array}\right) \\
& \left|\mathrm{B}_{\mathrm{n}} \mathrm{A}_{\mathrm{n}}\right|=\sqrt{{A n^{2}+B_{n}{ }^{2}}^{2}} \\
& =\sqrt{\begin{array}{l}
\left(\left(x_{A 0}+v_{a x .} n \Delta t\right)-\left(x_{B 0}+v_{B x .} n \Delta t\right)\right)^{2}+ \\
\left(\left(y_{A 0}+v_{a y} . n \Delta t\right)-\left(y_{B 0}+v_{B y} . n \Delta t\right)\right)^{2}
\end{array}}
\end{aligned}
$$

\section{B. Good Link Availability}

Once link life time has been determined, the next step is to get the availability of each link which will be termed as Good Link Availability (GLA).

Availability of a link to be good, or GLA, 


$$
P(G)=1-\frac{D_{(t)}}{T x_{\text {range }}}
$$

where $\mathrm{D}_{(\mathrm{t})}$ is the distance of the two nodes at the time of $\mathrm{t}$, while $T x_{\text {range }}$ is the transmission range of the two nodes, assumed to be the same. This equation is valid for $\mathrm{D}<\mathrm{T}$ and that $\mathrm{P}(\mathrm{G})=0$ for $\mathrm{D}>=\mathrm{T}$.

\section{Good Route Availability}

When the need to re-route occurs, the alternative route is selected based on the next available route with a good reliability of sustaining the route and this is called Good Route Availability (GRA). Before this happens, the existing route should have already been able to predict which other route has the highest availability of being able to sustain route life time over a certain period of time.

Fig. 4 shows an example of links and routes in a VANET. Assuming that node L2-1 is the source node and L2-4 is the destination, there are many possible routes available for this link, some of these possible routes are listed down below:

route 1: L2-1 > L2-2 > L2-3 > L2-4

route 2: L2-1 > L1-1 > L1-2 > L1-3>L1-4 > L2-4

route 3: L2-1 > L2-2 > L3-2 > L2-3>L 3-3 > L2-4

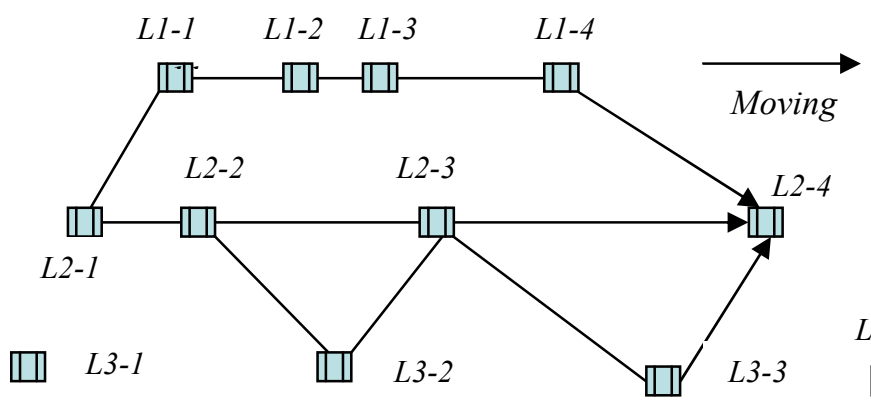

Fig. 4 Routing example to illustrate Good Route Availability

For the purpose of describing the theory, lets assume that route 1 is the current route. When travelling at a medium speed the vehicles are moving and there is a strong tendency that link L2-3 > L2-4 will break. Each link in a route knows its GLA and calculates the availability of the link to be alive within the stipulated speed ( P(A) ). Therefore the GRA for each route can be obtained by getting the product of the availability of each link in the route.

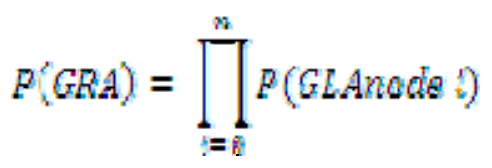

\begin{tabular}{|c|c|c|c|}
\hline Route & Links in Route & Sum & P(Gra) \\
\hline 2 & $L 2-1>L 1-1>L 1-2>$ & $0.95 * 0.88 * 0.77 *$ & 0.3724 \\
& $L 1-3>L 1-4>L 2-4$ & $0.65 * 0.89$ & \\
& & & \\
\hline 3 & $L 2-1>L 2-2>L 3-2>$ & $0.99 * 0.79 * 0.88 *$ & 0.4769 \\
& $L 2-3>L 3-3>L 2-4$ & $0.77 * 0.90$ & \\
\hline
\end{tabular}

The source node has the information of GRA for each neighbouring node and chooses the route with the highest GRA for re-routing. In this example, route 3 has the highest availability 0.4769 and this then can be chosen for re-routing. Note that the number of hops is the same and hence the two routes cannot be distinguished in this way.

\section{THE IMPLEMENTATION FRAMEWORK}

The implementation of the proposed functions can be classified into 3 main processes:

\section{A. Process I: Identifying and Passing Node Information}

Firstly it is necessary to pass information between nodes regarding their speed and velocity. The calculations of location predictions and probabilities for link and route stabilities are calculated once for each route based on the initial position and speed. Therefore, the information is gathered, passed and processes following the issuance of the first REPP packet from the destination node as it finalizes the route and this is done with a new packet called Route Measurement packet (RMEA) as shown in Fig 5.

The packet adapts the format of RREP packet as it also L3-4 avigates the same reverse route. The additional fields are the position, speed, and availability.

\begin{tabular}{|c|c|c|}
\hline \multicolumn{3}{|c|}{ 32-bit Flags } \\
\hline \multicolumn{3}{|c|}{ Destination IP address } \\
\hline \multicolumn{3}{|c|}{ Destination Sequence Number } \\
\hline \multicolumn{3}{|c|}{ Originator IP address } \\
\hline \multicolumn{3}{|c|}{ Longitude } \\
\hline \multicolumn{3}{|c|}{ Latitude } \\
\hline $\mathrm{v}_{\mathrm{x}}$ & $\mathrm{v}_{\mathrm{y}}$ & Accumulated Availability \\
\hline
\end{tabular}

Fig. 5 RMEA packet format

\section{B. Process II: Calculating Link and Route Probabilities}

At each intermediate node, the receiving node calculates the Good Link Availability (GLA) of the two nodes in that link and subsequently update the accumulated availability value 
as a product until it reaches the $\mathrm{SN}$ with the total product for availability of each link in the route in which at SN the product value is the Good Routing Availability (GRA). This process is shown in Fig. 6.



Fig. 6 RMEA packet process from destination to source node

\section{Process III: Assigning Pre-emptive Actions}

As the RREPs traverse their way back to the source node, the values of initial positions and speeds are collected and received by the source node for the computation of when a link is predicted to break and thus effecting the route. Instead of the standard process of RERR being issued after a link breaks, a pre-emptive action is taken before a link is predicted to break.

In the proposed system, the process is targeted for a case where there is a tendency of a link within a route to break while in transmission due to the movement of nodes, and also there are a few alternative routes available for a pre-emptive re-routing. The general process is shown in Fig. 7 which also differentiate the new and the standard process, and also the possible integration of the new to the existing process.

\section{SIMULATION AND ANALYSIS}

This section provides a set of simulations which show how the proposed method involving the estimation of link life time can be verified. The simulation was carried out using NS2 simulation software [13] which assumed that one unit of $\mathrm{x}$ and $\mathrm{y}$ is equal to one metre, and the transmission range is 150 metres. The details on the simulation parameters are shown in Fig. 8.

There are 5 sets of simulations with nodes in the range of 3 to 7 which are randomly distributed. The aim is to show the results in various situations, with only one node moving in one pair of transmission using FTP. In each simulation only one node is moving horizontally to indicate that there is no change of lane in the case of VANETs. The simulations cannot be claimed as a complete validation of the method, however they do provide some confidence of the validity of the technique.

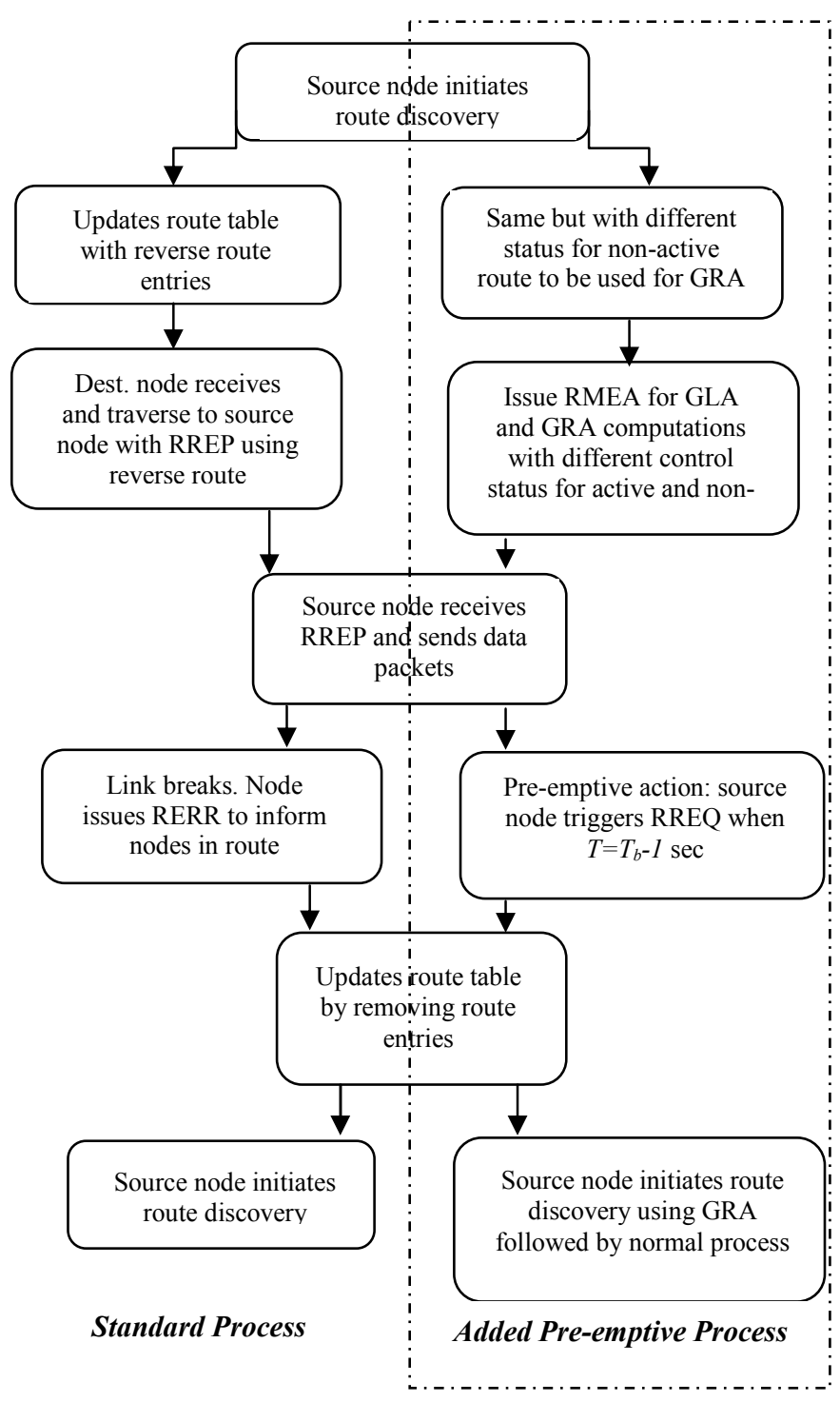

Fig. 7 A comparison of the standard and new process

One of the benefits of having the ability to predict locations and the time a link would break is to be able to calculate the time taken to do a re-route and the amount of data lost during the process. The data is obtained from the NS2 trace file where by referring to the calculated time the measurement is taken exactly with the difference when the first packet dropped and the first packet resumed.

The data involved during that period is then counted where there are 2 types of packets involved which are the TCP data packets and the control packets. The latter type consists of TCP acknowledgement packets (ACK), the AODV control 
packets, and the CBK packets. In NS2, CBK is referred to as the MAC call-back which indicates that the MAC layer was not able to transmit the packet and is therefore informing the upper routing layer about the transmission failure which usually is due to link failure.

Fig. 9 shows the time taken for the AODV routing protocol to do a re-route the data lost that can be avoided. The results range from 2-16 milliseconds for a 7-second simulation and the data involved are from 13- 2 Kbits. Although the results may not be linear with time it still manages to show quite a substantial amount of data can be lost without any preemptive actions.

\begin{tabular}{|l|l|}
\hline \multicolumn{1}{|c|}{ Parameters } & \multicolumn{1}{c|}{ Value } \\
\hline Wireless channel & Two-Ray Ground prop. model \\
\hline Media Access Control & MAC802.11 \\
\hline Antenna model & Omni-directional \\
\hline Topography dimension & $3500 x 670$ (in metres) \\
\hline Number of packets & $3-7$ \\
\hline Routing protocol & AODV \\
\hline Transmission range & 150 metres \\
\hline Node Speed & $25 \mathrm{~m} / \mathrm{s}$ \\
\hline Simulation time & 7 seconds \\
\hline Node movement & Cellular Automata \\
\hline Traffic Protocol & TCP \\
\hline
\end{tabular}

Fig. 8 Simulation Parameters

The fact is that AODV uses Hello packets to identify alternative links and routes. The proposed method would be able to avoid this as the function of GLA and GRA is to identify the best links and routes along with the time to do the pre-emptive re-routing.

\begin{tabular}{|c|c|c|}
\hline $\begin{array}{c}\text { Simulation } \\
\text { sets }\end{array}$ & $\begin{array}{c}\text { Re-route time } \\
(\mathbf{m} / \mathbf{s})\end{array}$ & $\begin{array}{c}\text { Data } \\
\text { Equivalent } \mathbf{( K b )}\end{array}$ \\
\hline 3-node & 4.472 & 17.911 \\
\hline 4-node & 8.032 & 22.302 \\
\hline 5-node & 16.472 & 18.973 \\
\hline 6-node & 9.012 & 24.434 \\
\hline 7-node & 2.672 & 13.594 \\
\hline
\end{tabular}

Fig. 9 Re-routing measurements

With a bigger picture in hand, for example in a highway situation, many link breakages can occur in a route. The approximate lost for each link break in recurring events can be avoided, and reducing the risk of dropped packets to happen. These are the two elements that can improve the network performance in VANETs.

\section{CONCLUSION}

By using the availability of each link and combining by multiplication provides a useful performance metric. In this paper the concept of GRA has been discussed and evaluated in a basic simulation. The other contribution of this paper is the inclusion of a suggested method of implementing this metric for the improvement of the AODV protocol.

\section{REFERENCES}

[1] Yousefi'., S., Mousavi, M. S., and Fathy, M. 2006. Vehicular Ad hoc Networks (VANETs): Challenges and Perspectives. In the 6th International Conference on ITS Telecommunications Proceedings. 761766. IEEE. Chengdu, China.

[2 J. Macker and M. S. Corson, "Mobile Ad hoc Network and the IETF," Association of Computing Machinery, vol. 2, pp. 9-14, 1998

[3] S. J. Lee, J. Hsu, R. Hayashida, M. Gerla and R. Bagrodia, "Selecting a Routing Strategy for Your Ad Hoc Network," Elseiver Computer Communications, vol. 26, pp. 723-733, 2003

[4] C. E. Perkins and E. M. Royer, "Ad-hoc on-demand distance vector routing," in Proceedings of the Second IEEE Workshop on Mobile Computer Systems and Applications 1999, pp. 90

[5] T. Goff, N. B. Abu-Ghzaleh, D. S. Phatak, R. Kahvecioglu, "Preemptive Routing in Ad Hoc Networks," Journal of Parallel and Distributed Computing - Special issue on Routing in mobile and wireless ad hoc networks, vol. 63, Issue 2, pp 123-140, Feb. 2003

[6] H. Menouar, M. Lenardi, F. Filali, "Improving Proactive Routing in VANETs with the MOPR Movement Prediction Framework," in the Proceedings of $7^{\text {th }}$ International Conference on ITS, Sophia Antipolis, France, 6 pp., June 2007

[7] S. Perur, A. Abhilash, S. Iyer, "Router Handoff: A Preemptive Route Repair Strategy for AODV," in the Proceedings of IEEE International Conference on Personal Wireless Communications, New Delhi, India, pp 168-171, 2002

[8] H. Q. Vo, C. S. Hong, "Hop-Count Based Congestion-Aware Multipath Routing in Wireless Mesh Network," in the Proceedings of International Conference on Information Networking, Busan, Korea, pp 1-5, Jan 2008

[9] F. Klemm, Z. Ye, S. V. Krishnamurthy, S. K. Tripathi, "Improving TCP Performance in ad hoc networks using signal strength based link management," Elsevier Ad Hoc Networks Journal, vol. 3, pp 175 191, March 2005

[10] J. Chen, X. Meng, Y. Guo, S. Grumbach, H. Sun, "Modeling and Predicting Future Trajectories of Moving Objects in a Constrained Network," in the Proceedings of the 7th International Conference on Mobile Data Management, Nara, Japan, pp 156-163, May 2006

[11] L. Chao, H. Aiqun, "Reducing the Message Overhead of AODV by Using Link Availability Prediction," in the Proceedings of Third International Conference on Mobile Ad-Hoc and Sensor Networks, MSN 2007, Beijing, China, pp. 113-122, December, 2007

[12] A.B. McDonald, T. Znati, "A Path Availability Model for Wireless AdHoc Networks," in the Proceedings of The IEEE Wireless Communications and Networking Conference (WCNC), New Orleans, LA, pp. 35-40, September 1999

[13] Information Sciences Institute University of Southern California. The Network Simulator NS-2. 2006. URL http://www.isi.edu/index.php 